\title{
Evaluating the biological functions of the prognostic genes identified by the Pathology Atlas in bladder cancer
}

\author{
YUANBIN CHEN $^{1 *}$, TING XU $^{2 *}$, FEI XIE $^{1}$, LIPING WANG $^{1}$, ZHIJUAN LIANG $^{1}$, DAN LI $^{1}$, \\ YE LIANG $^{1}$, KAIDONG ZHAO ${ }^{1}$, XIANGJIE $\mathrm{QI}^{3}$, XUECHENG YANG ${ }^{1}$ and WEI JIAO ${ }^{1}$ \\ ${ }^{1}$ Department of Urology, The Affiliated Hospital of Qingdao University, Qingdao 266003; \\ ${ }^{2}$ Department of Geratology, The 971th Hospital of PLA Navy, Qingdao 266000; ${ }^{3}$ Department of Urology, \\ People's Hospital of Linzi District, Zibo, Shandong 255400, P.R. China
}

Received May 25, 2020; Accepted September 17, 2020

DOI: $10.3892 /$ or.2020.7853

\begin{abstract}
The prognosis-associated genes of urinary bladder cancer have been systematically investigated in the Pathology Atlas project based on The Cancer Genome Atlas data. However, the biological functions of most genes in bladder cancer remain unknown. The present study investigated the biological function of 12 of the most significant survival-associated genes (ABRACL, MITD1, ZNF524, EMP1, HSPB6, CXorf38, TRIM38, ZNF182, ZNF195, SPRN, PTPN6 and LIPT1) in urothelial cancer reported by the Pathology Atlas project, with respect to cell proliferation and migration. In vitro, proliferation and migration analyses of T24 cells were performed following the transfection of the 12 prognostic genes. The results were validated with a small interfering (si)RNA library. Immunohistochemistry (IHC) analysis of clinical samples was performed to determine the association between gene expression and tumor metastasis. Furthermore, RNA sequencing was used to investigate the downstream signals. Among the 12 prognostic genes, MIT-domain containing protein 1 (MITDI) transfection was demonstrated to inhibit T24 cell migration to a certain degree. Experiments performed with a 7-gene siRNA library demonstrated that MITDI knockdown markedly upregulated cell migratory abilities. Mechanistically, the influence of MITD1 on cell signal transduction was assessed via RNA sequencing. Cell migration-associated genes, including KISSI, SPANXB1, SPINT1, PIWIL2, SNAI1, APLN and CTHRC1 were dysregulated. IHC analysis demonstrated that MITD1 protein expression was notably lower in metastatic lymph
\end{abstract}

Correspondence to: Dr Wei Jiao or Dr Xuecheng Yang, Department of Urology, The Affiliated Hospital of Qingdao University, 16 Jiangsu Road, Qingdao 266003, P.R. China

E-mail: jiaowei3929@163.com

E-mail:m18661805062@163.com

*Contributed equally

Key words: prognostic genes, migration, Pathology Atlas, bladder cancer nodes compared with the primary tumors. Taken together, the results of the present study suggest that the prognostic gene, MITD1 may serve as a migration inhibitor, and be developed as a potential therapeutic target for improving the prognosis of bladder cancer.

\section{Introduction}

Urothelial cancer is derived from the urothelium of the pelvis, ureter, bladder and urethra (1). The vast majority of urothelial cancer is bladder urothelial cancer, which accounts for $\sim 90 \%$ of all cases of bladder cancer (2). Bladder cancer is more common in men than women, with $\sim 549,393$ new cases and 199,922 mortalities worldwide, annually (3). The 5-year survival rate of patients with bladder cancer is $\sim 80 \%$ (2), and it has not improved in the last three decades compared with several other malignant tumors, such as prostate cancer, lung cancer, kidney cancer and breast cancer (2). Investigating the genes that determine the survival outcome of patients and attempting to block or activate the associated pathways may help improve the therapeutic efficacy.

The Pathology Atlas project (4) was performed based on the large open-access transcriptome data and the clinical metadata of The Cancer Genome Atlas (TCGA, https://www.genome. gov/Funded-Programs-Projects/Cancer-Genome-Atlas) and the Human Protein Atlas databases (http://www.proteinatlas. org). A systems-level approach was used to assess the transcriptome of 17 major types of cancer, including urothelial cancer, with respect to clinical outcome, in which several survival-associated genes were identified. However, whether the prognostic genes serve any biological roles in cancer development remains largely unknown.

Uncontrolled cell proliferation is one of the basic characteristics of cancer, while cancer metastasis is considered a major cause of cancer associated mortality (5). Previous studies have demonstrated that the genes that regulate cancer cell proliferation or migration rate also influence patient survival $(6,7)$. The present study investigated the biological function of 12 of the most significant survival-associated genes (ABRACL, MITD1, ZNF524, EMP1, HSPB6, CXorf38, TRIM38, ZNF182, ZNF195, SPRN, PTPN6 and LIPT1) in urothelial cancer reported by the Pathology Atlas project, with 
respect to cell proliferation and migration. For the MIT-domain containing protein 1 (MITD1) gene, which regulates cell migration in the present study, the downstream molecules were assessed via RNA sequencing. Immunohistochemistry (IHC) analysis was performed to compare the expression levels of MITD1 between primary tumors and metastatic cancer cells, in order to determine whether MITDl is involved in cancer metastasis in vivo.

\section{Materials and methods}

Searching the prognostic genes. The prognostic genes were identified as follows: The home page of the Human Protein Atlas database (https://www.proteinatlas.org/humanproteome/pathology) was opened and 'urothelial cancer' under the 'correlation analysis and prognostic genes' section was selected. There are 1,092 prognostic genes in the online table. A gene is considered prognostic by the Pathology Atlas if correlation analysis of gene expression (FPKM) and prognosis (survival) resulted in Kaplan-Meier plots with high significance $(\mathrm{P}<0.001)(4)$. Here, the $\mathrm{P}$-values were calculated with the best expression cut-offs. Based on the cut-off value of each gene, patients were classified into two groups (high expression group and low expression group) and the association between survival and gene expression was assessed. The best expression cut-off refers to the FPKM value that yields maximal difference with regards to survival between the two groups at the lowest log-rank P-value (4).

In addition to the P-values calculated with the best expression cut-offs, the Pathology Atlas also provides the P-values with the median expression cut-offs. Median expression refers to the median FPKM value calculated based on the gene expression data from all patients in this dataset (4). This information is attained as follows: i) The web page of each gene in the table is opened, ii) the median expression value under the 'urothelial cancer-interactive survival scatter plot \& survival analysis' section is selected and the Kaplan-Meier curve and $\mathrm{P}$-value are adjusted to provide results based on the median expression.

The candidate genes for investigation were screened by initially selecting 29 genes with $\mathrm{P}<1 \times 10^{-6}$ (best expression cut-off). A total of six genes with $\mathrm{P} \geq 0.01$ (median expression cut-off) were excluded from the final analysis. Among the remaining 23 genes, five genes [ZNF224 (8), CCNL1 (9), $A P O B E C 3 H$ (10), ANAPC4 (11) and IKBKB (12)] were further excluded as they have previously been reported to be associated with tumor development. Cloning primers were designed for the remaining 18 genes (Table SI). Following PCR amplification, restriction digestion ligation and transformation, 12 genes (ABRACL, MITD1, ZNF524, EMP1, HSPB6, CXorf38, TRIM38, ZNF182, ZNF195, SPRN, PTPN6 and LIPT1) were successfully cloned into the pcDNA3.1+ eukaryotic expression vector (Invitrogen; Thermo Fisher Scientific, Inc.).

Clinical samples for IHC. A total of 10 patients with bladder cancer from the Affiliated Hospital of Qingdao University were enrolled in the present study between December 2016 and June 2018. The patients had not received radiotherapy or chemotherapy prior to surgery and the pathological type is urothelial cancer. The primary tumors, adjacent normal tissues (the distance between the tumor tissues and normal tissues $>3 \mathrm{~cm}$ ) and metastatic lymph nodes were surgically removed by radical cystectomy and pelvic lymphadenectomy, and immediately fixed in $10 \%$ formalin at room temperature for $24 \mathrm{~h}$ and embedded in paraffin. The present study was approved by the Human Ethics Committee of The Affiliated Hospital of Qingdao University (Qingdao, China; approval no. QYFYWZLL25948). The present study was performed in accordance with the latest version of the Declaration of Helsinki (13). Written informed consent was provided by all patients prior to the study start. The detailed clinical information of each patient is listed in Table I.

Vector constructing. The gene coding sequences of $A B R A C L$, MITD1, ZNF524, EMP1, HSPB6, CXorf38, TRIM38, ZNF182, ZNF195, SPRN, PTPN6 and LIPT1 were amplified via PCR. PCR thermocycling conditions were as follows: $98^{\circ} \mathrm{C}$ for $1 \mathrm{~min}$ and 30 cycles of $98^{\circ} \mathrm{C}$ for $10 \mathrm{sec}, 55^{\circ} \mathrm{C}$ for $15 \mathrm{sec}$, and $72^{\circ} \mathrm{C}$ for $30 \mathrm{sec}$. PrimeSTAR MAX polymerase (cat. no. R045A; Takara Biomedical Technology (Beijing), Co., Ltd.) was used and the DNA of T24 cell was the template. The products were subsequently cloned into a pcDNA3.1+ vector (Invitrogen; Thermo Fisher Scientific, Inc.) with double digestion and ligation. Following transformation of the ligation products into DH5 $\alpha$ competent cells (Takara Biomedical Technology, Co., Ltd.), respectively, the bacteria were cultured using the spread plate method (14) and 10 bacterial colonies for each gene were validated via PCR. The PCR products of positive colonies were sent to Sanger sequencing (BGI, Inc.) and the sequence compare was performed using Chromas software v2.4.1 (http://technelysium. com.au/wp/chromas) for the sequence correctness. The primer sequences used for cloning are listed in Table SI.

DNA and small interfering (si)RNA transfection. Transfection was performed at room temperature for $6 \mathrm{~h}$. The T24 and 5637 bladder tumor cell lines (cat. no. SCSP-536 and TCHu 1; Chinese Academy of Sciences Cell Bank) were seeded into 12-well plates at a density of 100,000 cells/well the day before transfection. For transfection with the gene expression constructs, $1 \mu \mathrm{g}$ of the gene expression vector (constructed by ourselves) and $2 \mu \mathrm{l}$ p3000 reagent (Invitrogen; Thermo Fisher Scientific, Inc.) were diluted into Opti-MEM solution (Invitrogen; Thermo Fisher Scientific, Inc.) and mixed with equal volumes of Opti-MEM, which contained $1.5 \mu$ l Lipofectamine ${ }^{\circledR} 3000$ reagent (Invitrogen; Thermo Fisher Scientific, Inc.). Following incubation at room temperature for $5 \mathrm{~min}$, the 12 gene mixtures were added into the appropriate cell cultures, respectively. With regards to the siRNA transfection of genes ABACL, CXof38, LIPT1, MITD1, ZNF524, TRIM38 and EHBP1, $30 \mathrm{pmol}$ siRNA or negative control (NC) oligonucleotide was diluted into Opti-MEM solution and mixed with equal volumes Opti-MEM, which contained $3 \mu$ Lipofectamine ${ }^{\circledR} 3000$ reagent. Cells were collected by trypsinization for the following experiments (15), $48 \mathrm{~h}$ post-transfection. The siRNAs and NC oligonucleotide (cat. no. siN0000002-1-5) were produced by Guangzhou RiboBio, Co., Ltd., and the siRNA targets of the assessed genes are presented in Table SII.

MTT assay. Cells that underwent transfection were reseeded into 96-well culture plates at a density of 2,000 cells/well, 
Table I. Clinical information of patient samples for immunohistochemistry.

\begin{tabular}{rclcl}
\hline Patient number & Age, years & \multicolumn{1}{c}{ Sex } & Differentiation & \multicolumn{1}{c}{ Sample site } \\
\hline 1 & 67 & Male & Poor & Normal tissue, Primary tumor, Lymph node metastasis \\
2 & 77 & Female & Poor & Normal tissue, Primary tumor, Lymph node metastasis \\
3 & 55 & Male & Poor & Normal tissue, Primary tumor, Lymph node metastasis \\
4 & 60 & Male & Poor & Lymph node metastasis \\
5 & 64 & Male & Poor & Primary tumor, Lymph node metastasis \\
6 & 75 & Male & Poor & Primary tumor, Lymph node metastasis \\
7 & 73 & Female & Poor & Normal tissue, Primary tumor, Lymph node metastasis \\
8 & 70 & Male & Poor & Primary tumor, Lymph node metastasis \\
9 & 75 & Male & Poor & Lymph node metastasis \\
10 & 64 & Male & Poor & Primary tumor, Lymph node metastasis \\
\hline
\end{tabular}

and maintained in $100 \mu 1$ RPMI-1640 medium (Invitrogen; Thermo Fisher Scientific, Inc.) supplemented with $10 \%$ fetal bovine serum (FBS; Gibco, Thermo Fisher Scientific, Inc.) at $37^{\circ} \mathrm{C}$ in $5 \% \mathrm{CO}_{2}$. The wells in the borders of the plates were filled with phosphate buffered saline (PBS; Beyotime Institute of Biotechnology) to protect against the influence of culture medium volatilization. Cells were incubated with $10 \mu 1$ MTT reagent (Beyotime Institute of Biotechnology) at $37^{\circ} \mathrm{C}$ for $4 \mathrm{~h}, 72 \mathrm{~h}$ post-transfection. Following the MTT incubation, the purple formazan crystals were dissolved using $100 \mu \mathrm{l}$ dimethyl sulfoxide (Beyotime Biotechnology) and viability was subsequently analyzed at a wavelength of $490 \mathrm{~nm}$ using a spectrophotometer (Thermo Fisher Scientific, Inc.) $(16,17)$. All experiments were performed in triplicate.

Transwell assay. The Transwell assay was performed as previously described (18). Cells that underwent transfection were washed twice with RPMI-1640 medium without FBS. A total of $1 \times 10^{5}$ cells were resuspended in RPMI-1640 medium without FBS and plated in the upper chambers of Transwell plates (cat. no. 353097; BD Biosciences), which had transparent polyester membranes and a pore size of $8 \mu \mathrm{m}$. RPMI-1640 medium supplemented with 10\% FBS was plated in the lower chambers. Following incubation at $37^{\circ} \mathrm{C}$ for $12 \mathrm{~h}$, the upper chambers were fixed with $100 \%$ methyl alcohol at room temperature for $10 \mathrm{~min}$ and subsequently stained with $0.1 \%$ crystal violet at room temperature for $10 \mathrm{~min}$, and the excess dye was washed off with water. The unmigrated cells were removed using cotton swabs. Stained cells were counted in five randomly selected fields using a light microscope (magnification, x100) (18).

Wound healing assay. T24 cells were seeded into 6-well culture plates at a density of $1 \times 10^{6}$ cells/well the day before transfection. Cells transfected with the negative control and MITD1 siRNAs were further cultured in PRMI-1640 medium supplemented with $10 \% \mathrm{FBS}$ at $37^{\circ} \mathrm{C}$ for $48 \mathrm{~h}$. Cells were scratched with $200 \mu 1$ pipette tips, washed once with PBS, and further incubated in serum-free medium at $37^{\circ} \mathrm{C}$ cell incubator for $48 \mathrm{~h}$. The scratches were observed at 0,24 and $48 \mathrm{~h}$, respectively, using an inverted light microscope (magnification, x100; IX73; Olympus Corporation). The results were quantified using ImageJ software (version 1.50i; National
Institutes of Health) and analyzed using GraphPad Prism software (version 6; GraphPad Software, Inc.). All experiments were performed in triplicate.

IHC staining. IHC analysis was performed as previously described (19). Briefly, the surgically removed tissues were fixed in $10 \%$ formalin at room temperature for $24 \mathrm{~h}$ and embedded in paraffin. Paraffin-embedded tissue samples were cut into $4-\mu \mathrm{m}$-thick sections and heated overnight at $50^{\circ} \mathrm{C}$. Prior to immunostaining, deparaffinization and hydration were performed in xylene and a descending ethanol series (100, 95 and $75 \%$ ). Heat Induced Epitope Retrieval was performed by heating the slides immersed in retrieval solution: $10 \mathrm{mM}$ sodium citrate buffer, $\mathrm{pH} 6.0$, with $1 \mathrm{mM}$ EDTA, at $125^{\circ} \mathrm{C}$ for $4 \mathrm{~min}$ in a pressure boiler. Tissue sections were incubated with $0.3 \%$ (v/v) $\mathrm{H}_{2} \mathrm{O}_{2}$ in $95 \%$ ethanol at room temperature for $5 \mathrm{~min}$ to inhibit endogenous peroxidase activity. Tissue sections were washed twice with PBS. Immunohistochemical staining was performed with Polymer HRP Detection System for Rabbit Primary Antibody (cat. no. PV-6001; ZSGB-BIO, Inc.). All incubations were performed at room temperature and reagents were applied at a volume of $200 \mu$ l per slide. According to the instruction manual, blocking was not necessary due to the special design of the primary antibody dilution buffer. The slides were incubated with MITD1 antibody (cat. no. HPA036162; Atlas Antibodies AB; 1:50) at room temperature for $30 \mathrm{~min}$. The slides were rinsed three times with PBS, and subsequently incubated with peroxidase labeled polymer conjugated to goat anti-rabbit IgG antibody working solution (cat. no. PV-6001; ZSGB-BIO, Inc.) at room temperature for $30 \mathrm{~min}$. The slides were re-washed twice in wash buffer and developed at room temperature for 5 min using DAB (cat. no. PV-6001; ZSGB-BIO, Inc.) as the substrate. Counterstaining was performed using hematoxylin solution (cat. no. C0107; Beyotime Institute of Biotechnology) for $3 \mathrm{~min}$ at room temperature. Cells were observed under a light microscope (magnification $\mathrm{x} 400$ ).

RNA sequencing. RNA was extracted from cells that underwent transfection using TRIzol ${ }^{\circledR}$ reagent (Invitrogen; Thermo Fisher Scientific, Inc.), according to the manufacturer's protocol. The library construction, sequencing and data analysis were performed by Annoroad Gene Technology Corporation 
(http://en.annoroad.com), with an Illumina Sequencing platform (HiSeq2500; Illumina, Inc.). Sequencing libraries were generated using the NEBNext ${ }^{\circledR}$ Ultra $^{\mathrm{TM}}$ RNA Library Prep kit for Illumina ${ }^{\circledR}$ (cat. no. E7530L; NEB, Inc. https://www.neb.com), according to the manufacturer's instructions. RNA integrity and concentration were assessed using the RNA Nano 6000 Assay kit of the Bioanalyzer 2100 system (cat. no. 5067-1511; Agilent Technologies, Inc.). The concentration of library was measured using the Qubit ${ }^{\circledR}$ RNA Assay kit (cat. no. Q32852; Thermo Fisher Scientific, Inc.) to preliminarily quantify and subsequently dilute to $1 \mathrm{ng} / \mu \mathrm{l}$. The accurate concentration was measured using a StepOnePlus ${ }^{\mathrm{TM}}$ Real-Time PCR System (Thermo Fisher Scientific, Inc.). The valid concentrations of the libraries are greater than $10 \mathrm{nM}$. The clustering of the index-coded samples was performed on a cBot cluster generation system using the HiSeq PE Cluster kit v4-cBot-HS (cat. no. PE-401-4001; Illumina, Inc.), according to the manufacturer's instructions. Following cluster generation, the libraries were sequenced on an Illumina platform (HiSeq2500; Illumina, Inc.) and 150-bp paired-end raw reads were generated.

RNA sequencing analysis. The Perl script was used to filter the original data to guarantee the data quality. The steps were as follows: i) Trim Smart-seq2 public primer sequence from the reads; ii) remove the contaminated reads of adapters; iii) remove the low quality reads and iv) remove the reads with $\mathrm{N}$ base $>5 \%$ for total bases. The reference genomes and the annotation file were downloaded from ENSEMBL database (http://www. ensembl.org/index.html). Bowtie2 v2.2.3 (http://bowtie-bio. sourceforge.net/bowtie2/index.shtml) was used to build the genome index, and Clean Data was subsequently aligned to the reference genome using HISAT2 v2.1.0 (http://ccb.jhu.edu/software/hisat2/faq.shtml). Reads Count for each gene in each sample was counted using HTSeq v0.6.0 (https://htseq.readthedocs.io), and Fragments Per Kilobase Millon Mapped Reads (FPKM) was subsequently calculated to estimate the expression level of genes in each sample. DESeq2 (http://www.bioconductor. org/packages/release/bioc/html/DESeq2.html) was used to compare differences in gene expression levels between groups. Genes with $\mathrm{P} \leq 0.05$ and fold change $(\mathrm{FC}) \geq 1.5$ were classified as differentially expressed genes (DEGs).

Enriched pathway analysis. Kyoto Encyclopedia of Genes and Genomes (KEGG) pathway enrichment analyses were performed using the Database for Annotation, Visualization and Integration Discovery (DAVID; version 6.8; https://david. ncifcrf.gov/home.jsp). DEGs between the control and MITDI knockdown groups were uploaded onto DAVID. The enriched KEGG pathways of the genes were assessed using the "functional annotation tool' within DAVID. Modified fisher exact P-value was calculated to determine the enriched extent. Gene count=2 was used as the threshold to include the items in the table.

Reverse transcription-quantitative $(R T-q) P C R$. Transfection efficiency was demonstrated via RT-qPCR. Total RNA were extracted from T24 and 5637 cells using TRIzol reagent (Invitrogen; Thermo Fisher Scientific, Inc.) and reverse transcribed at $37^{\circ} \mathrm{C}$ for 15 min using the PrimeScript ${ }^{\mathrm{TM}} \mathrm{RT}$ reagent kit with gDNA Eraser (cat. no. RR047; Takara Biomedical Technology, Inc.). qPCR was subsequently performed using
Platinum $^{\text {TM }}$ SYBR $^{\text {TM }}$ Green qPCR SuperMix-UDG (Thermo Fisher Scientific, Inc.), according to the manufacturer's protocol. GAPDH was used as the reference gene. The qPCR primers are listed in Table SIII. qPCR thermocycling conditions were as follows: $95^{\circ} \mathrm{C}$ for $1 \mathrm{~min}$ and 40 cycles of $95^{\circ} \mathrm{C}$ for $15 \mathrm{sec}, 60^{\circ} \mathrm{C}$ for $30 \mathrm{sec}$, and $72^{\circ} \mathrm{C}$ for $15 \mathrm{sec}$. All data were analyzed using the $2^{-\Delta \Delta \mathrm{Cq}}$ method (20).

Statistical analysis. Statistical analysis was performed using GraphPad Prism 6 (GraphPad Software, Inc.). Experiments were performed at least in triplicate, and data are presented as the mean \pm standard deviation. Unpaired Student's t-test was used to compare differences between two groups, while one-way ANOVA followed by Tukey's post hoc test was used to compare differences between multiple groups. $\mathrm{P}<0.05$ was considered to indicate a statistically significant difference.

\section{Results}

Expression vectors constructed for the survival-associated genes. In order to measure the function of the prognostic genes on cell proliferation and migration, the present study focused on the no bias system-level analysis of the Pathology Atlas, which is based on the genome-wide transcriptomics analysis of TCGA project and the Human Protein Atlas project (4) (http://www.proteinatlas.org). The present study selected the top 18 genes significantly associated with survival in patients with urothelial cancer in the Pathology Atlas project, which met the following criteria: i) The prognostic $\mathrm{P}<1 \times 10^{-6}$ (best cut-off); ii) the prognostic $\mathrm{P}<1 \times 10^{-2}$ (median expression cut-off) and iii) has rarely been reported to be associated with cancer progression. Following PCR amplification and restriction digestion, 12 genes (ABRACL, MITD1, ZNF524, EMP1, HSPB6, CXorf38, TRIM38, ZNF182, ZNF195, SPRN, PTPN6 and $L I P T 1)$ were successfully cloned into the pcDNA3.1+ eukaryotic expression vector (Fig. 1A). The process of screening the 12 genes is presented in Fig. 1B and detailed information of the 12 genes is presented in Table II. All 12 constructs were validated via Sanger sequencing.

Effects of the prognostic genes on tumor cell viability. Recombinant gene transfection may induce cell death or change cell proliferation rate (21). Thus, the present study assessed cell viability via the MTT assay $72 \mathrm{~h}$ post-transfection (Fig. 2A). The results demonstrated that in the in vitro experiments, there was no significant change in cell viability induced by transfection of the 12 gene expression constructors (Fig. 2B).

Screening migration-associated genes among the survival-associated genes. The 12 gene expression vectors were subsequently transfected into T24 bladder cancer cells, respectively. The migratory ability of cells was assessed via the Transwell assay, $48 \mathrm{~h}$ post-transfection. The results demonstrated that the migratory rate of cells transfected with CXorf38, EMP1, TRIM38, ZNF182 and ZNF524 did not changed compared with the mean value of the 12 overexpression groups. However, ABRACL, PTPN6, SPRN and ZNF195 transfection increased the cell migratory ability. The three genes, including two favorable-survival associated genes (MITDI and LIPT1) and one unfavorable-survival 
Table II. Information on the 12 prognostic genes.

\begin{tabular}{|c|c|c|c|c|}
\hline Gene & Gene description & $\begin{array}{l}\text { Prognostic } \\
\text { function }\end{array}$ & $\begin{array}{c}\text { P-value (Best } \\
\text { expression cut-off) }\end{array}$ & $\begin{array}{l}\text { P-value (Median } \\
\text { expression cut-off) }\end{array}$ \\
\hline ABRACL & ABRA C-terminal like & Favorable & $1.65 \times 10^{-6}$ & $1.69 \times 10^{-5}$ \\
\hline CXorf38 & Chromosome $\mathrm{X}$ open reading frame 38 & Favorable & $1.44 \times 10^{-8}$ & $7.54 \times 10^{-7}$ \\
\hline EMP1 & Epithelial membrane protein 1 & Unfavorable & $9.69 \times 10^{-8}$ & $8.63 \times 10^{-5}$ \\
\hline HSPB6 & Heat shock protein, alpha-crystallin-related, B6 & Unfavorable & $1.43 \times 10^{-6}$ & $9.51 \times 10^{-6}$ \\
\hline LIPT1 & Lipoyltransferase 1 & Favorable & $9.60 \times 10^{-8}$ & $9.71 \times 10^{-5}$ \\
\hline MITD1 & $\begin{array}{l}\text { MIT, microtubule interacting and transport, } \\
\text { domain containing } 1\end{array}$ & Favorable & $5.46 \times 10^{-8}$ & $2.00 \times 10^{-6}$ \\
\hline PTPN6 & Protein tyrosine phosphatase, non-receptor type 6 & Favorable & $2.64 \times 10^{-8}$ & $2.41 \times 10^{-7}$ \\
\hline SPRN & Shadow of prion protein homolog (zebrafish) & Favorable & $1.58 \times 10^{-7}$ & $3.42 \times 10^{-4}$ \\
\hline TRIM38 & Tripartite motif containing 38 & Favorable & $4.94 \times 10^{-9}$ & $7.93 \times 10^{-6}$ \\
\hline ZNF182 & Zinc finger protein 182 & Favorable & $1.55 \times 10^{-7}$ & $7.99 \times 10^{-3}$ \\
\hline ZNF195 & Zinc finger protein 195 & Favorable & $4.79 \times 10^{-7}$ & $2.92 \times 10^{-5}$ \\
\hline ZNF524 & Zinc finger protein 524 & Favorable & $4.98 \times 10^{-8}$ & $4.73 \times 10^{-4}$ \\
\hline
\end{tabular}
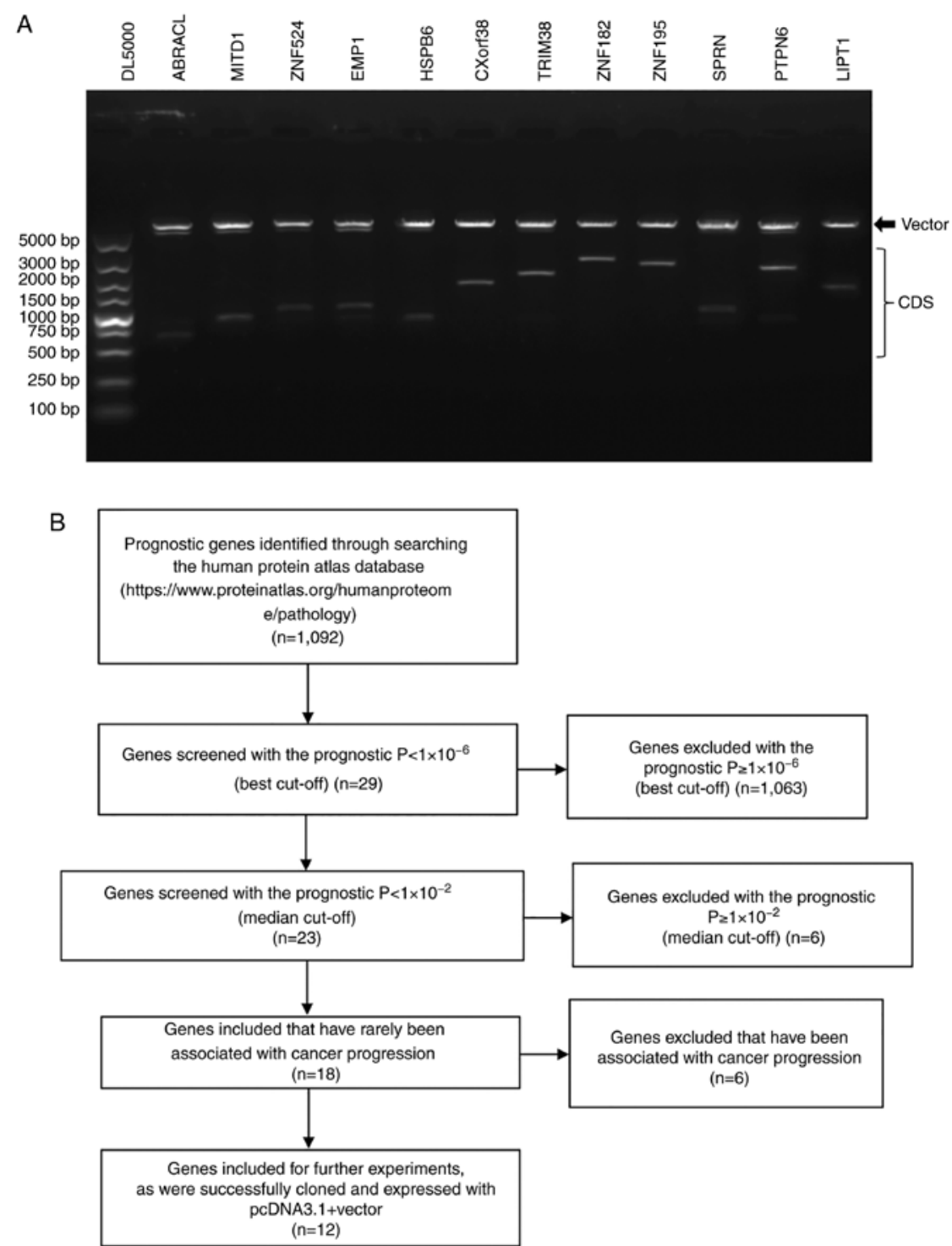

Figure 1. Expression vectors constructed for the survival-associated genes. (A) Restrictive enzymatic map of the 12 gene expression constructs. Line 1, DL5000 maker. Lines 2-13, the constructs were digested with corresponding restriction enzymes. For each line of the 12 constructs, the upper band is the digested vector and the lower band is the inserted gene CDS. (B) Flow diagram outlining the selection process. CDS, coding sequence; bp, base pair. 

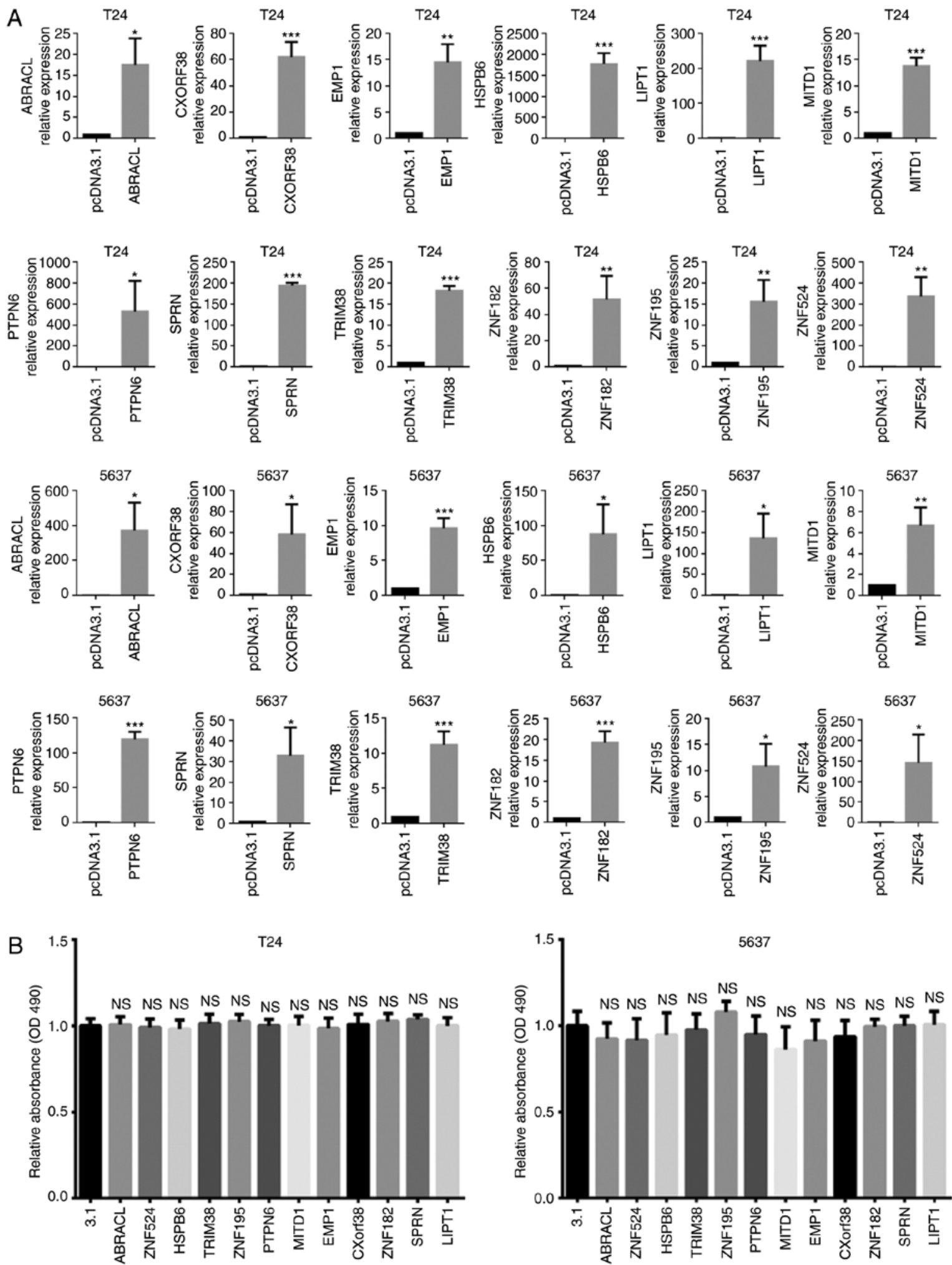

Figure 2. Effects of the prognostic genes overexpression on cell viability of bladder cancer cells in vitro. (A) The effects of overexpression were validated via reverse transcription-quantitative PCR. (B) The results of the MTT assay. The cells and the gene expression vectors used for transfection were indicated in the figure. All experiments were repeated at least for three times. The data are presented as the mean \pm standard deviation. " $\mathrm{P}<0.05 ;{ }^{* * *} \mathrm{P}<0.01{ }^{* * * *} \mathrm{P}<0.001 \mathrm{vs}$. control group (pcDNA3.1). NS, no significance; MITD1, MIT-domain containing protein 1; 3.1, pcDNA3.1+ vector.

associated gene, $H S P B 6$, inhibited cell migration at a certain level (Fig. 3).

A siRNA library of 7 genes, in which each gene had two siRNAs, was used to screen the genes regulating cell migration with a loss-of-function strategy (Fig. 4A).
MITD1 knockdown markedly increased cell migration (Fig. 4B and C). The wound healing assay was performed to validate the role of MITD1 on cell migration and the results demonstrated that MITD1 knockdown significantly promoted cell migration (Fig. 5). 
A T24

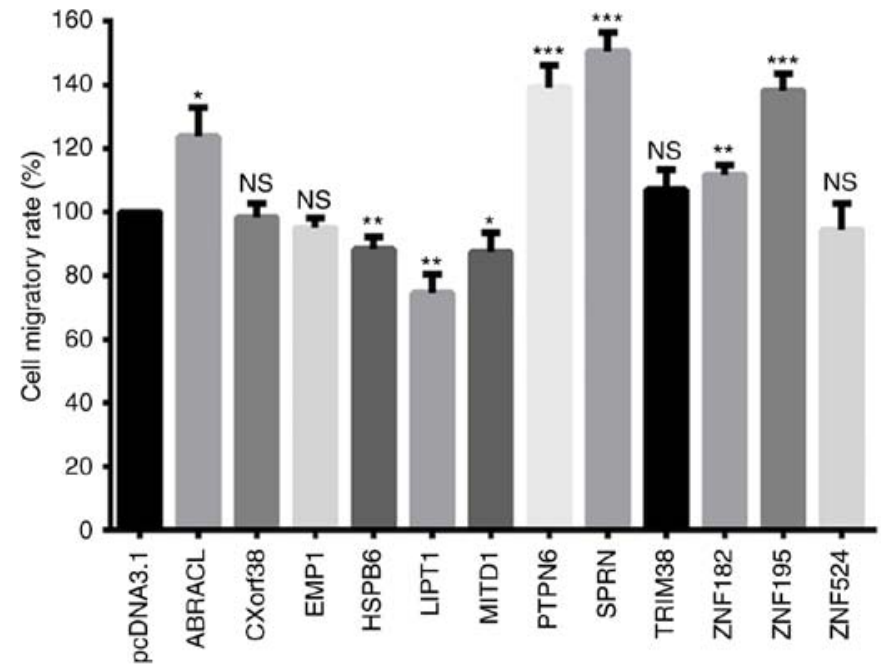

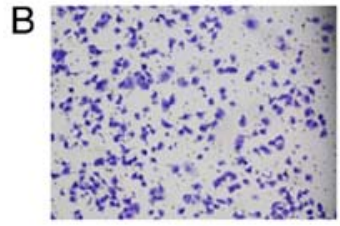

pcDNA3.1

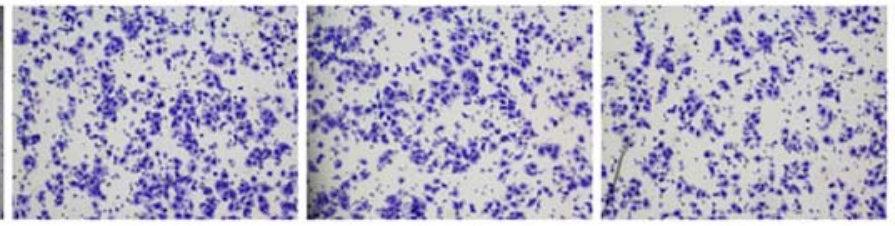

ABRACL

CXorf38 EMP1

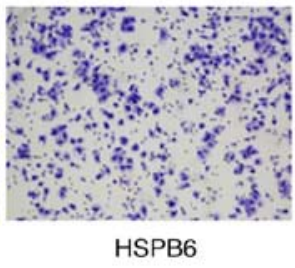

HSPB6
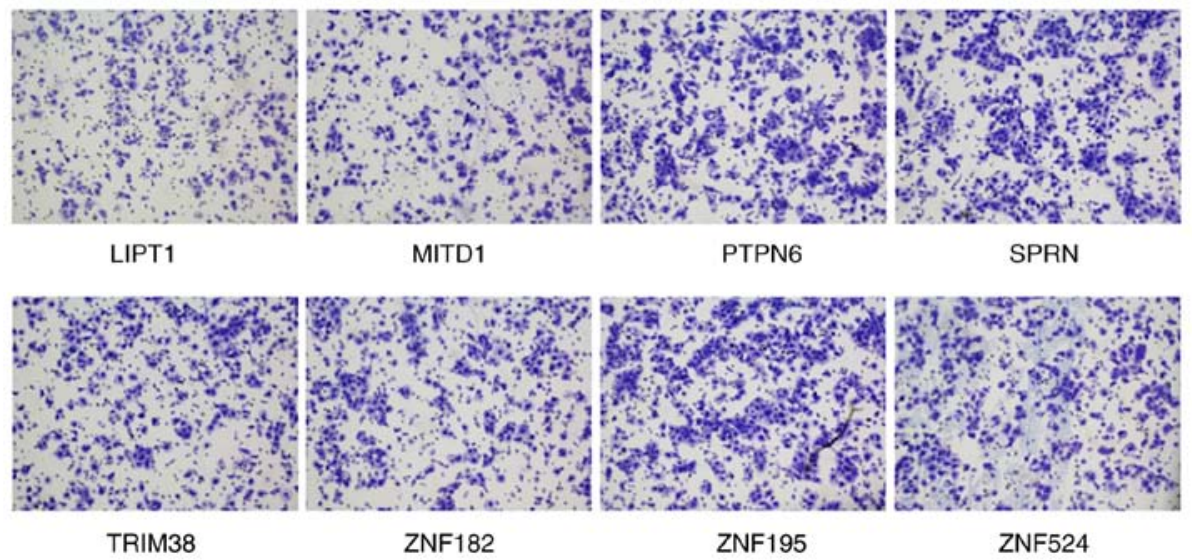

Figure 3. Effects of the 12 genes on cell migratory ability detected via the Transwell assay. (A) The statistical data of the Transwell assay. The cell and the gene expression vectors used for transfection were indicated in the figure. (B) Representative images of Transwell assay. $\mathrm{P}<0.05$; ${ }^{* *} \mathrm{P}<0.01 ;{ }^{* * *} \mathrm{P}<0.001$ vs. control group (pcDNA3.1). NS, no significance; MITD1, MIT-domain containing protein 1.

MITD1 downstream molecule analysis. As MITD1 inhibited cell migration in vitro, downstream genes were investigated with RNA sequencing. T24 cells were transfected with MITD1 siRNAs or NC oligonucleotide, while the untreated cells were cultured as the BLANK group. Cell RNA was extracted using TRIzol ${ }^{\circledR}$ reagent $48 \mathrm{~h}$ post-transfection, and transcriptome sequencing was subsequently performed. The results demonstrated that cells transfected with MITD1 siRNA exhibited dysregulation of the migration associated genes, KISS1, SPANXB1, SPINT1, PIWIL2, SNAII, APLN, EDNI and CTHRCl compared with the NC and BLANK groups (Fig. 6). The enriched KEGG pathways were analyzed using DAVID Bioinformatics Resources 6.8. The results demonstrated that 'transcriptional dysregulation in cancer' was significantly enriched $(\mathrm{P}=0.019)$ (Table SIV).

MITD1 expression is downregulated in metastatic tumors. Lymphatic metastasis is one of the main characteristics underlying bladder cancer progression, and a key predictor of cancer-specific survival (22). To identify the associations between MITD1 and lymphatic metastasis, the present study assessed MITD1 protein expression in the primary tumors and paired metastasis in lymph nodes. IHC analysis demonstrated that there was a significant decrease in MITD1 protein expression in the metastatic tumors compared with the primary tumors (mean IHC score, 6.875 vs. 2.800; P<0.001; Fig. 7).

\section{Discussion}

Improving tumor prognosis remains a major challenge. Based on the genome-wide transcriptomic data of TCGA and the clinical metadata, the Pathology Atlas project performed a systematical survival analysis of 17 major cancer types, and identified the most significant survival-associated genes (4). However, the biological function and molecular mechanisms 

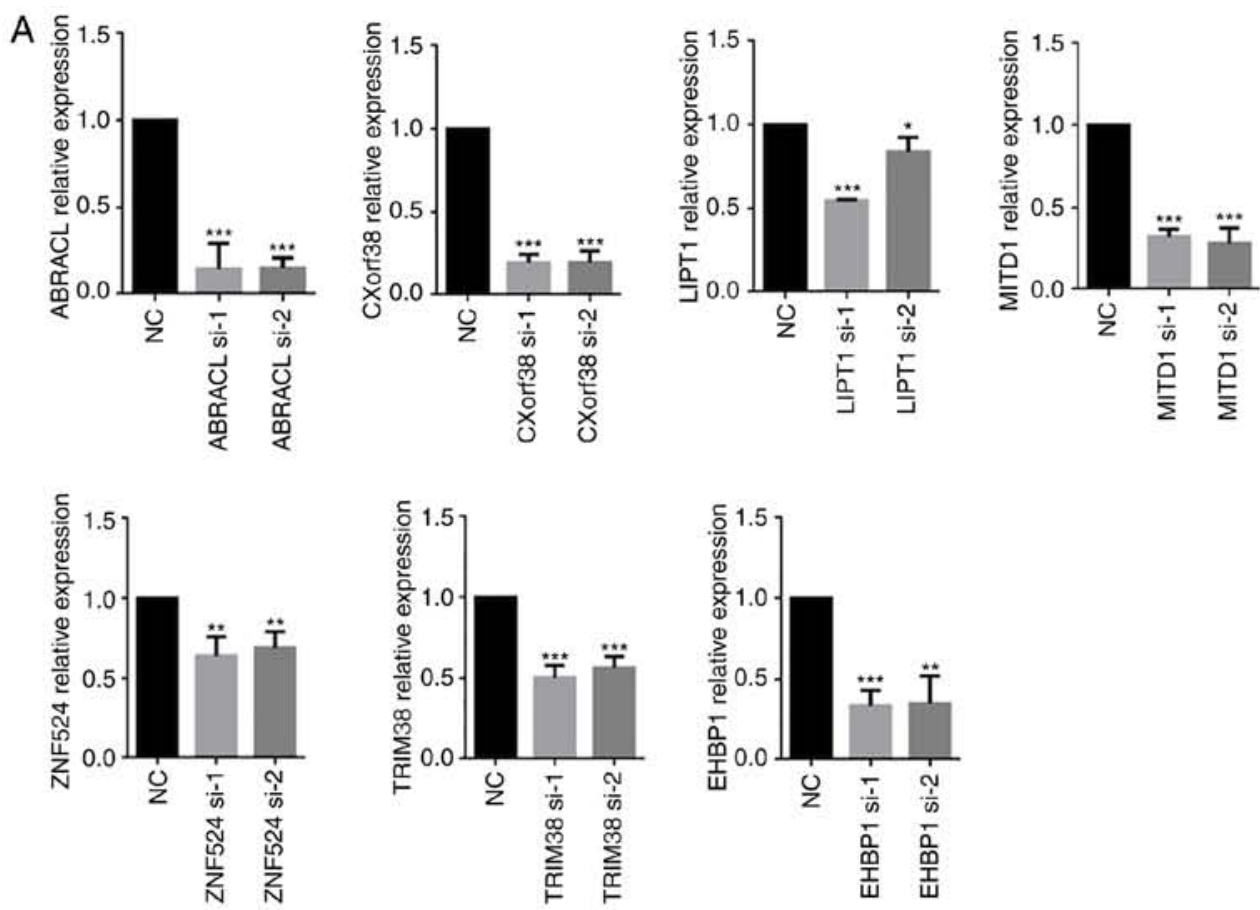

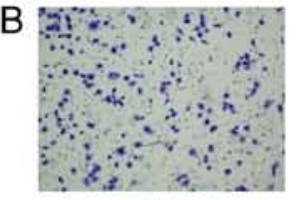

NC

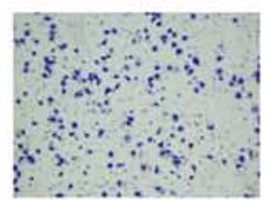

LIPT1 si-1

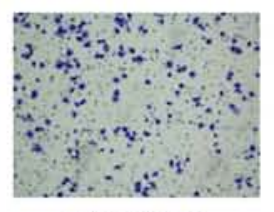

ZNF524 si-2

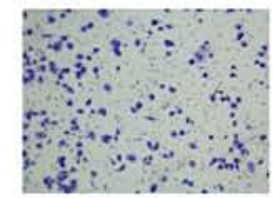

ABRACL si-1

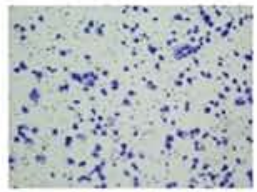

LIPT1 si-2

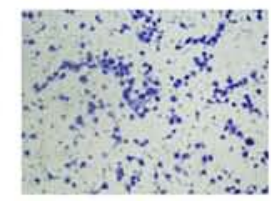

TRIM38 si-1

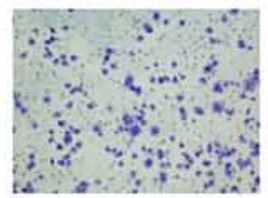

ABRACL Si-2

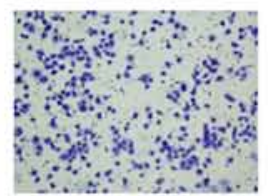

MITD1 si-1

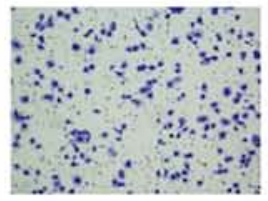

TRIM38 si-2

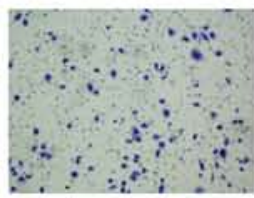

CXort38 si-1

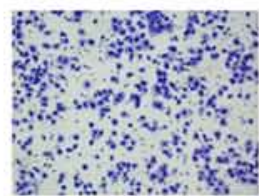

MITD1 si-2

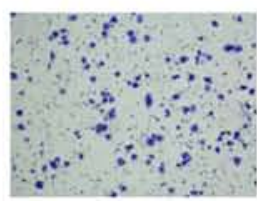

EHBP1 si-1

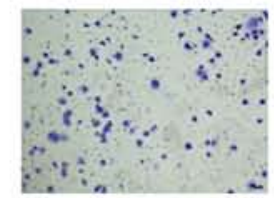

CXort38 si-2

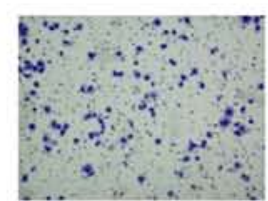

ZNF524 si-1

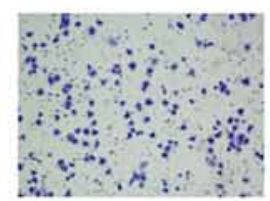

EHBP1 si-2

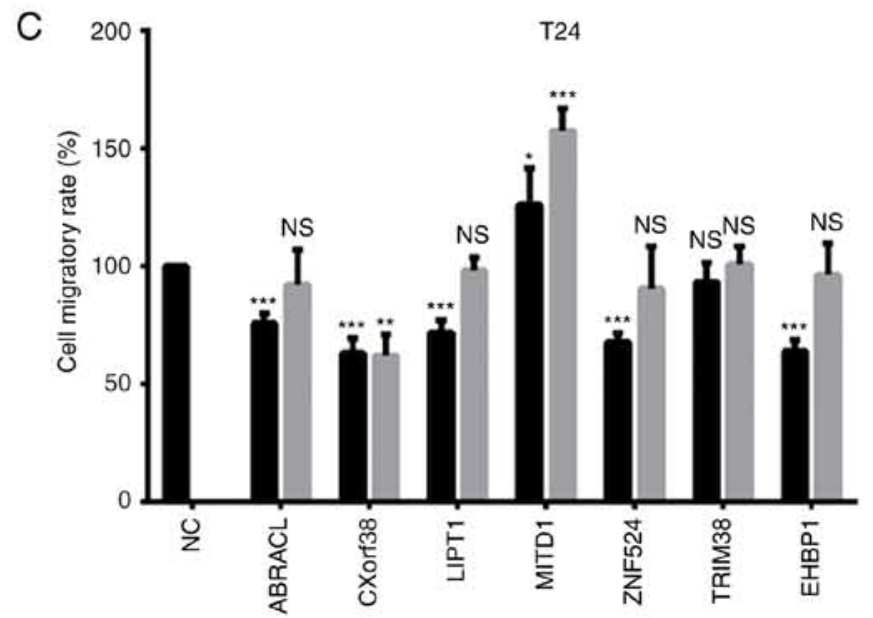

Figure 4. Effects of the seven genes on cell migratory ability detected with the Transwell assay. (A) Transfection with siRNA was validated via reverse transcription-quantitative PCR analysis. (B) Representative images. (C) Statistical analysis of the Transwell assay. The cell and siRNAs used for transfection were indicated in the figure. Data are presented as the mean \pm standard deviation. ${ }^{*} \mathrm{P}<0.05 ;{ }^{* * *} \mathrm{P}<0.01 ;{ }^{* * * *} \mathrm{P}<0.001$ vs. control group (NC). si, small interfering; NS, no significance; NC, negative control oligonucleotide; MITD1, MIT-domain containing protein 1. 
A
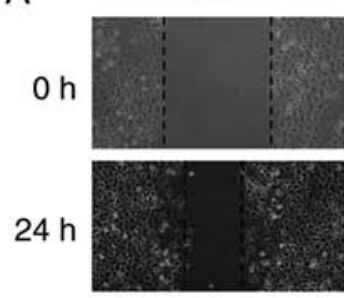

$48 \mathrm{~h}$

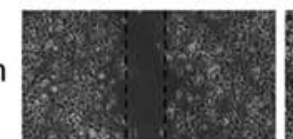

MITD1 Si-1
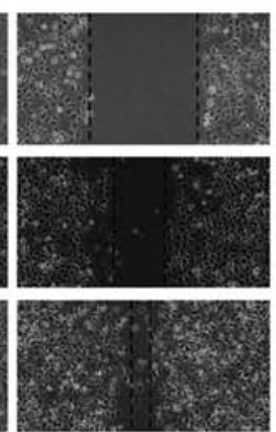

MITD1 Si-2

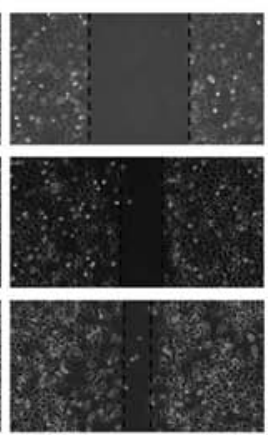

B

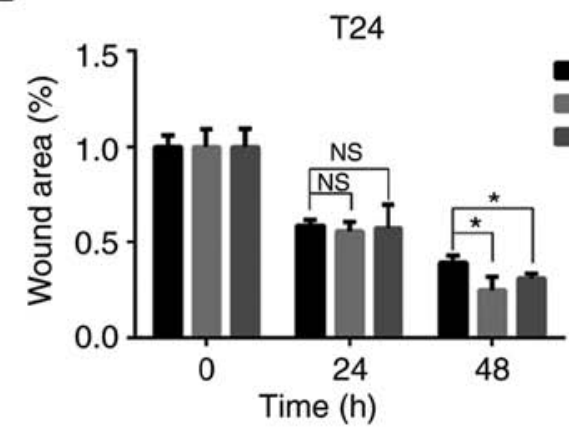

Figure 5. MITD1 knockdown promotes cell wound healing. (A) Representative images of the wound healing assay. T24 cells transfected with siRNAs were scratched and photographed at 0,24 and $48 \mathrm{~h}$ (magnification, x100). (B) Statistical analysis performed relative to the starting wound area at $0 \mathrm{~h}$. Data are presented as the mean \pm standard deviation. ${ }^{*} \mathrm{P}<0.05$. si, small interfering; NS, no significance; NC, negative control oligonucleotide; MITD1, MIT-domain containing protein 1.

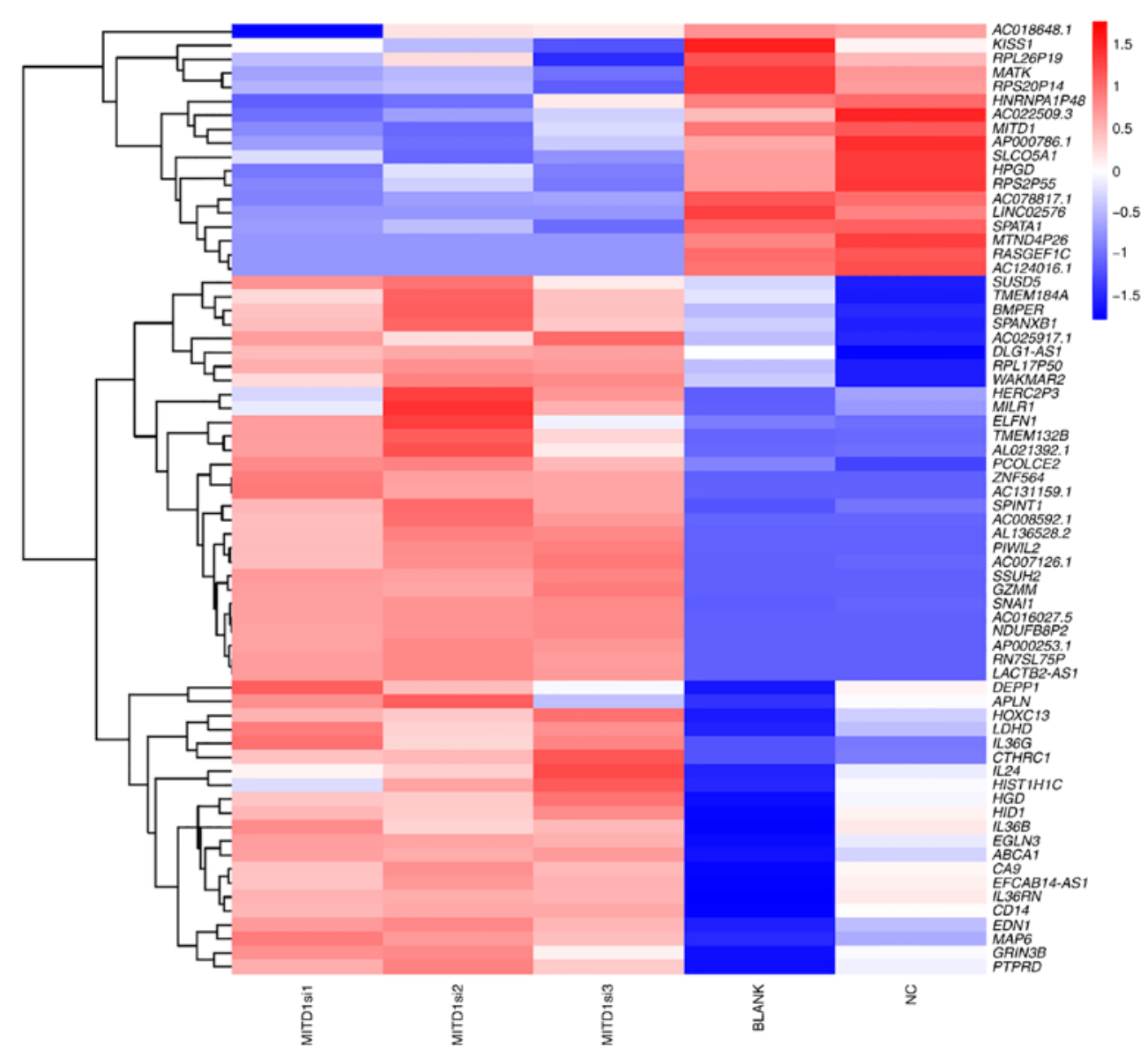

Figure 6. Heatmap of DEGs following MITD1 knockdown. The rows represent the DEGs of RNA sequencing. The genes in bold are metastasis-associated genes. The columns represent the samples with different treatment. BLANK cells were untreated. NC cells were treated with control oligonucleotide. Hierarchical clustering was used for classification. Blue-white-red gradient indicates relative expression levels of each gene. Blue indicates low expression level and red indicates high expression level. DEGs, differentially expressed genes; NC, negative control; si, small interfering; MITD1, MIT-domain containing protein 1.

of these genes has not yet been identified. The present study investigated the function of prognostic genes identified in the Pathology Atlas project with in vitro experiments, with respect to cell proliferation and migration rate. Among the 12 prognostic genes, a favorable gene, MITDl exhibited a notable effect on cell migration. When exogenous recombined MITDI was transfected into T2 4 cells, the cell migratory ability was downregulated, while knockdown of MITDl promoted cell migration. Using RNA sequencing, the present study also revealed several potential downstream molecules of MITD1 to mediate migration arrest. To validate the function of MITDI on cell migration in vivo, the present study compared the MITD1 protein level in primary tumors and lymph node metastatic tumors. The results demonstrated that MITD1 expression decreased in metastatic tumors, which was in accordance with the in vitro results. Taken together, these results suggest that MITD1 may be a migration inhibitor and a potential therapeutic target to improve bladder cancer prognosis. 

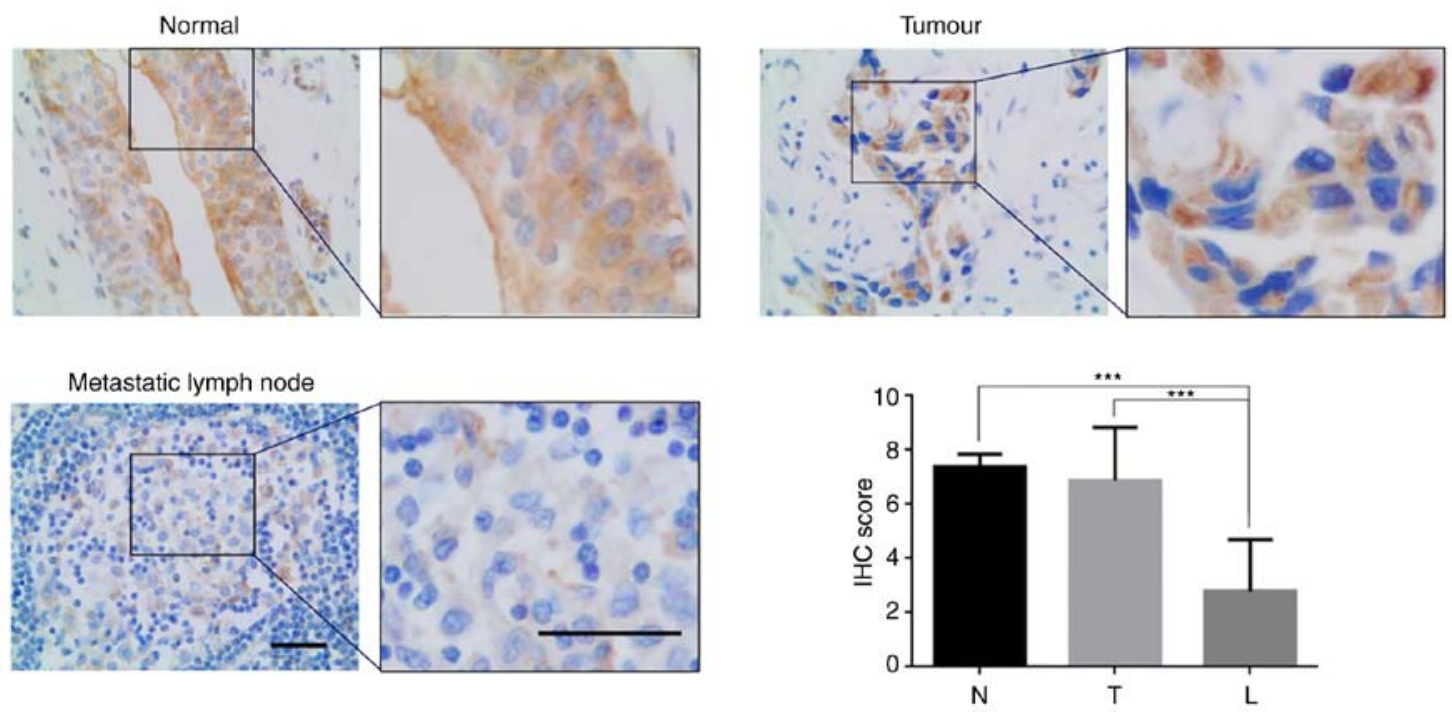

Figure 7. IHC analysis of MITD1 in bladder cancer. Upper left, representative IHC images of normal bladder tissues. Upper right, representative IHC images of tumors. Lower left, representative IHC images of metastatic lymph nodes. The notable parts of each image are enlarged on the right. Scale bars, $50 \mu \mathrm{m}$. Lower right, statistical data of the IHC experiment. Data are presented as the mean \pm standard deviation. Results were analyzed via one-way ANOVA followed by Tukey's post hoc test. ${ }^{* * *} \mathrm{P}<0.001$. IHC, immunohistochemistry; N, normal bladder tissue; T, tumor; L, metastatic lymph node.

MITD1 interacts with endosomal sorting complexes required for transport III (ESCRT-III) and may be recruited to the midbody to participate in cytokinesis $(22,23)$. In the present study, both gain- and loss-of-function in vitro experiments demonstrated that MITDI may be a migration inhibitor, which is consistent with the favorable role on survival identified in the Pathology Atlas project.

To determine the molecular mechanism underlying MITDl-induced inhibition of cell migration, RNA sequencing was performed. The results demonstrated that several potential downstream molecules (KISS1, SPANXB1, SPINT1, PIWIL2, SNAI1, APLN, EDN1 and CTHRC1), which have been reported to be associated with cell metastasis, were dysregulated following MITD1 knockdown. For example, the EMT regulator, SNAII was significantly upregulated, while the metastasis suppressor KISSI (24-26) was downregulated to a certain degree.

Furthermore, although the sample size was limited, the present study demonstrated that MITD1 expression levels were much higher in the primary tumors compared with the metastatic tumors. Collectively, these results suggest that tumor clones with lower expression levels of MITD1 may be advantageous for metastasis in vivo.

Uncontrolled cell proliferation is one of the hallmarks of cancer, and it is widely accepted that the cell proliferation rate may be involved in cancer progression, and affect the prognosis (5). However, the present study did not observe any notable effects of the prognostic gene overexpression on cell viability. Neither serious cell cytotoxicity or accelerated proliferation were introduced following transfection of these genes. These results suggest that the genes may influence tumor development independent of altering the cell proliferation rate. Conversely, the same level of cell viability was used in each transfection group to ensure that the results of the migration assay were not affected by the differences in cell cytotoxicity or proliferation.

With regards to the consistency between the in vitro experiments and clinical survival studies, 10 of the 12 prognostic genes (ABRACL, ZNF524, EMP1, HSPB6, CXorf38, TRIM38, ZNF182, ZNF195, SPRN and PTPN6) exhibited the unexpected role on cell migration in the overexpression studies. In the Pathology Atlas project, the high expression levels of ABRACL,CXorf38, PTPN6, SPRN,TRIM38, ZNF182,ZNF195 and ZNF524 were associated with favorable survival (4). However, the results of the present study demonstrated that overexpression of these genes did not weaken cell migratory ability compared with overexpression of EMPI and HSPB6, high expression of which is associated with poor survival outcomes (4). There were three possible reasons for this: i) The majority of the prognostic genes identified by the Pathology Atlas project may act as a 'passenger' for tumor development, not the 'driver'; ii) the in vitro experiments may not completely mimic the authentic in vivo context and iii) the prognostic genes may promote cancer progress by altering other characteristics, such as invasion, immune escape and angiogenesis.

One of the limitations of the present study is that the detailed molecular mechanisms by which MITD1 regulates cell migration and influences patient survival were not investigated. The detailed molecular mechanisms will be investigated by in vivo research in the future.

The present study investigated the role of the prognostic genes identified in the Pathology Atlas project on cell proliferation and migration, and demonstrated that the favorable prognostic gene, MITDl may inhibit cell migration and metastasis, which implies a novel promising target for targeted therapies of bladder cancer.

\section{Acknowledgements}

Not applicable.

\section{Funding}

The present study was supported by the National Natural Science Foundations of China (grant nos. 81772713, 81472411, 
81981260351 and 81972378), the Taishan Scholar Program of Shandong Province (grant no.tsqn20161077), the Natural Science Foundation of Shandong Province (grant no. ZR2016HQ18) and the Key Research and Development Program of Shandong Province (grant no. 2018GSF118197).

\section{Availability of data and materials}

The datasets used and analyzed during the present study are available from the corresponding author upon reasonable request. The RNA-seq raw FASTQ files were deposited in the NCBI SRA database (accession no. PRJNA658813).

\section{Authors' contributions}

YC, WJ and XY designed the present study. YC, TX and XQ drafted the initial manuscript. YC, TX, WJ, FX and LW performed the cell experiments. ZL, YL, KZ, XQ and DL contributed to statistical analysis and designed the tables and figures. WJ, XY and XQ were involved in project management and supervised the study. WJ and XY contributed to revising the manuscript for intellectual content and language editing. All authors read and approved the final manuscript.

\section{Ethics approval and consent to participate}

The present study was approved by the Human Ethics Committee of The Affiliated Hospital of Qingdao University (Qingdao, China; approval no. QYFYWZLL25948). Written informed consent was provided by all patients prior to the study start.

\section{Patient consent for publication}

Not applicable.

\section{Competing interests}

The authors declare that they have no competing interests.

\section{References}

1. Miyazaki J and Nishiyama H: Epidemiology of urothelial carcinoma. Int J Urol 24: 730-734, 2017.

2. Berdik C: Unlocking bladder cancer. Nature 551: S34-S35, 2017.

3. Bray F, Ferlay J, Soerjomataram I, Siegel RL, Torre LA and Jemal A: Global cancer statistics 2018: GLOBOCAN estimates of incidence and mortality worldwide for 36 cancers in 185 countries. CA Cancer J Clin 68: 394-424, 2018.

4. Uhlen M, Zhang C, Lee S, Sjöstedt E, Fagerberg L, Bidkhori G, Benfeitas R, Arif M, Liu Z, Edfors F, et al: A pathology atlas of the human cancer transcriptome. Science 357: eaan2507, 2017.

5. Fouad YA and Aanei C: Revisiting the hallmarks of cancer. Am J Cancer Res 7: 1016-1036, 2017.

6. Zhang D, Jin N, Sun W, Li X, Liu B, Xie Z, Qu J, Xu J, Yang X, $\mathrm{Su} \mathrm{Y}$, et al: Phosphoglycerate mutase 1 promotes cancer cell migration independent of its metabolic activity. Oncogene 36 : 2900-2909, 2017.

7. Xue Y, Xiao H, Guo S, Xu B, Liao Y, Wu Y and Zhang G Indoleamine 2,3-dioxygenase expression regulates the survival and proliferation of Fusobacterium nucleatum in THP-1-derived macrophages. Cell Death Dis 9: 355, 2018.

8. Harada Y, Kanehira M, Fujisawa Y, Takata R, Shuin T, Miki T, Fujioka T, Nakamura Y and Katagiri T: Cell-permeable peptide DEPDC1-ZNF224 interferes with transcriptional repression and oncogenicity in bladder cancer cells. Cancer Res 70: 5829-5839, 2010.
9. Sticht C, Hofele C, Flechtenmacher C, Bosch FX, Freier K, Lichter P and Joos S: Amplification of Cyclin L1 is associated with lymph node metastases in head and neck squamous cell carcinoma (HNSCC). Br J Cancer 92: 770-774, 2005.

10. Starrett GJ, Luengas EM, McCann JL, Ebrahimi D, Temiz NA, Love RP, Feng Y, Adolph MB, Chelico L, Law EK, et al: The DNA cytosine deaminase APOBEC3H haplotype I likely contributes to breast and lung cancer mutagenesis. Nat Commun 7: 12918, 2016.

11. Eifler K, Cuijpers SAG, Willemstein E, Raaijmakers JA, El Atmioui D, Ovaa H, Medema RH and Vertegaal ACO: SUMO targets the APC/C to regulate transition from metaphase to anaphase. Nat Commun 9: 1119, 2018.

12. Carneiro-Lobo TC, Scalabrini LC, Magalhães LDS, Cardeal LB, Rodrigues FS, Dos Santos EO, Baldwin AS, Levantini E, Giordano RJ and Bassères DS: IKK $\beta$ targeting reduces KRAS-induced lung cancer angiogenesis in vitro and in vivo: A potential anti-angiogenic therapeutic target. Lung Cancer 130: 169-178, 2019.

13. World Medical Association: World Medical Association Declaration of Helsinki: Ethical principles for medical research involving human subjects. JAMA 310: 2191-2194, 2013.

14. Sanders ER: Aseptic laboratory techniques: Plating methods. J Vis Exp: e3064, 2012.

15. Yan S, Tang Z, Chen K, Liu Y, Yu G, Chen Q, Dang H, Chen F, Ling J, Zhu L, et al: Long noncoding RNA MIR31HG inhibits hepatocellular carcinoma proliferation and metastasis by sponging microRNA-575 to modulate ST7L expression. J Exp Clin Cancer Res 37: 214, 2018.

16. Liu Y, Zeng C, Bao N, Zhao J, Hu Y, Li C and Chi S: Effect of Rab23 on the proliferation and apoptosis in breast cancer. Oncol Rep 34: 1835-1844, 2015.

17. Huang W, Chen C, Liang Z, Qiu J, Li X, Hu X, Xiang S, Ding X and Zhang J: AP-2 $\alpha$ inhibits hepatocellular carcinoma cell growth and migration. Int J Oncol 48: 1125-1134, 2016.

18. Zhou B, Wang GZ, Wen ZS, Zhou YC, Huang YC, Chen Y and Zhou GB: Somatic mutations and splicing variants of focal adhesion kinase in non-small cell lung cancer. J Natl Cancer Inst 110, 2018.

19. Sun Y,Luo J, Chen Y, Cui J,Lei Y, Cui Y, Jiang N, Jiang W, Chen L, Chen Y, et al: Combined evaluation of the expression status of CD155 and TIGIT plays an important role in the prognosis of LUAD (lung adenocarcinoma). Int Immunopharmacol 80: 106198, 2020.

20. Livak KJ and Schmittgen TD: Analysis of relative gene expression data using real-time quantitative PCR and the 2(-Delta Delta C(T)) method. Methods 25: 402-408, 2001.

21. Tian ZQ, Xu YZ, Zhang YF, Ma GF, He M and Wang GY: Effects of metallothionein-3 and metallothionein-1E gene transfection on proliferation, cell cycle, and apoptosis of esophageal cancer cells. Genet Mol Res 12: 4595-4603, 2013.

22. Kassouf W, Svatek RS, Shariat SF, Novara G, Lerner SP, Fradet Y, Bastian PJ, Aprikian A, Karakiewicz PI, Fritsche HM, et al: Critical analysis and validation of lymph node density as prognostic variable in urothelial carcinoma of bladder. Urol Oncol 31: 480-486, 2013.

23. Hadders MA, Agromayor M, Obita T, Perisic O, Caballe A, Kloc M, Lamers MH, Williams RL and Martin-Serrano J: ESCRT-III binding protein MITD1 is involved in cytokinesis and has an unanticipated PLD fold that binds membranes. Proc Natl Acad Sci USA 109: 17424-17429, 2012.

24. Lee JH and Welch DR: Suppression of metastasis in human breast carcinoma MDA-MB-435 cells after transfection with the metastasis suppressor gene, KiSS-1. Cancer Res 57: 2384-2387, 1997.

25. Nash KT and Welch DR: The KISS1 metastasis suppressor: Mechanistic insights and clinical utility. Front Biosci 11: 647-659, 2006.

26. Lee JH, Miele ME, Hicks DJ, Phillips KK, Trent JM, Weissman BE and Welch DR: KiSS-1, a novel human malignant melanoma metastasis-suppressor gene. J Natl Cancer Inst 88: 1731-1737, 1996.

This work is licensed under a Creative Commons Attribution-NonCommercial-NoDerivatives 4.0 International (CC BY-NC-ND 4.0) License. 